\title{
Pulse transit time: Relationship to blood pressure
}

\author{
PAUL A. OBRIST, KATHLEEN C. LIGHT, JAMES A. McCUBBIN, \\ J. STANFORD HUTCHESON, and J. LEE HOFFER \\ Departments of Psychiatry and Anesthesiology, University of North Carolina Medical School \\ Chapel Hill, North Carolina 27514
}

\begin{abstract}
The relationships between pulse transit time (PTT) and both systolic (SBP) and diastolic blood pressure (DBP) were evaluated in 114 human subjects during rest and stress. PTT was found to covary quite consistently with SBP, but not with DBP, when blood pressure was measured both noninvasively and invasively. Pharmacological blockade of the sympathetic innervation of the heart appreciably attenuated the relationship between PTT and SBP.
\end{abstract}

A continuous, noninvasive, and nonencumbering ${ }^{1}$ method to measure variations in blood pressure has several applications in psychophysiology. For example, there is a need to assess blood pressure under field conditions when current technology with an auscultatory cuff is not feasible. One such measure is pulse transit time, PTT $^{2}$ (Gribbin, Steptoe, \& Sleight, 1976; Jernstedt \& Newcomer, 1974; Steptoe, Smylan, \& Gribbin, 1976; Weltman, Sullivan, \& Gredon, 1964; Williams \& Williams, 1965). The most definitive assessment of this index to date is that of Steptoe et al. (1976), who demonstrated an appreciable degree of covariation between PTT and mean blood pressure, ${ }^{3}$ as measured intra-arterially (i.e., as measured directly with an arterial catheter implanted within the artery). The studies reported here are intended to evaluate further this relationship, focusing on the systolic (SBP) and diastolic (DBP) blood pressure separately rather than on the mean arterial pressure. There is evidence to suggest that the SBP varies more consistently than the DBP with PTT (Weltman et al., 1964). This possibility is also consistent with the more recent observation that the mechanisms which control the SBP and DBP can vary relatively independently as a function of differences in the qualitative features among stressors (Obrist, Gaebelein, Teller, Langer, Grignolo, Light, \& McCubbin, 1978).

Six experiments have been performed as parts of our investigation of this measure. All involved measuring PTT and blood pressure while the subject rested and then during three stressors which earlier studies have shown can evoke appreciable changes in blood pressure (Obrist et al., 1978). Three of the studies differed only with respect to where the arterial pulse wave was transduced. These transduction sites were the carotid,

This research was supported by Research Grant MH 07995 , from the National Institute of Mental Health, and Grant HL 18976 and Postdoctoral Award F32-HL-05531, from the National Heart, Lung and Blood Institute, National Institutes of Health. temporal, and radial arteries. The intent of using the various transduction sites was to see if the distance the pulse wave travels from the heart influences the degree of covariation between PTT and blood pressure. A fourth study used only the carotid pulse wave, but in this case the sympathetic innervation to the heart was pharmacologically blocked. This was done to determine the degree of covariation when changes in myocardial force were minimized. In the last two studies, blood pressure was measured intra-arterially, using the radial pulse wave from the arterial catheter to obtain the PTT. These two studies differed in that the sympathetic innervation to the heart was intact in one study; in the other, it was pharmacologically blocked. Intra-arterial readings of blood pressure were used because they provide the most accurate means available to measure blood pressure and an artifact-free pulse wave. Thus, the results from this study can be compared to those from the previous four experiments in which blood pressure and PTT measures were obtained noninvasively.

\section{METHOD}

\section{Subjects}

The subjects were 114 healthy young adult males. Of these, 102 were undergraduates recruited from the introductory psychology course at the University of North Carolina, Chapel Hill. These undergraduates served in the first four studies. The remaining 12 were either graduate or medical students who served in the last two studies.

\section{Equipment and Method of Measurement}

Transit time was measured on each cardiac cycle as the time between the initiation of the pre-ejection period (as indicated by the $R$ wave of the EKG) and the peak of the pulse wave associated witn that cardiac cycle. The time was derived by specially constructed electronic circuitry ${ }^{4}$ which had a resolution to the nearest millisecond. When blood pressure was measured noninvasively, an automated cuff system was used which provides five to six SBP and DBP readings per minute (see Obrist et al., Note 1, for details). Heart rate and carotid dP/dt were also recorded (Obrist, Lawler, Howard, Smithson, Martin, \& Manning, 1974; Obrist et al., 1978). 


\section{Procedure}

The experimental stressors included the cold pressor which involves submerging the foot for $90 \mathrm{sec}$ in a bucket filled with ice and then water, an 8-min pornographic movie explicitly depicting "normal" heterosexual behavior between two adults, and an unsignaled (vigilance) reaction time shock avoidance task with shock avoidance and a monetary bonus contingent on performance time. The vigilance task had a duration of $14 \mathrm{~min}$. A 2- to 3-min resting baseline preceded each stressor, and a final minute followed the last (see Obrist et al., 1978, for details).

\section{Data Quantification}

When blood pressure was monitored noninvasively, 100 or more SBP and DBP readings were quantified along with the corresponding PTT, HR, and dP/dt values. Data were sampled from $2 \mathrm{~min}$ of each of the four rest periods, the entire $90 \mathrm{sec}$ of the cold pressor, the first $4 \mathrm{~min}$ of the film, and $8 \mathrm{~min}$ of the avoidance task. The latter included Minutes $1-4,8-9$, and 13-14. The consistency with which SBP and DBP covaried with PTT, HR, and carotid $\mathrm{dP} / \mathrm{dt}$ within each subject was evaluated with Pearson product-moment correlations. A similar analysis of the data was performed when blood pressure was monitored directly by selecting SBP or DBP values in a systematic manner over all conditions so that a total of 100 cardiac cycles were sampled. This selection process was intended to be similar to the analysis used when blook pressure was measured noninvasively. In addition, 100 consecutive cardiac cycles were quantified at the onset of each stressor, as well as an additional 100 cycles 5-6 min into the avoidance task. The correlations between PTT and each of the other cardiovascular measures were again obtained. Also, correlations were obtained when 25 additional resting values were included in the sample. This was done in order to obtain a greater range of values.

\section{RESULTS}

SBP was found to covary quite appreciably with PTT under both methods of measuring blood pressure when the sympathetic innervations to the heart were intact. On the other hand, the degree of covariation with DBP was appreciably less. Table 1 summarizes the median within-subject correlations for each experiment. The table indicates the median correlation as a function of pulse-wave recording site, the method of blood pressure measurement, and whether the sympathetic innervation of the heart was intact or blocked.

With regard to SBP when the innervations are intact, there is a trend for the correlations ${ }^{5}$ to be larger when the pulse wave is recorded further from the heart, that is, the radial pulse. Under this condition, inspection of the individual correlations from all three pulse-wave recording sites revealed that, of the 60 correlations, 58 were significant $(\mathrm{p}<.05)$, with 44 exceeding -.70 and 27 exceeding -.80 . There was also a trend for the magnitude of the correlation to be larger with individuals who demonstrated greater increases in SBP during the stressors. Finally, the effect of blocking the sympathetic innervation of the heart was to attenuate appreciably the magnitude of the correlations. Of the 18 subjects, the $r$ values were significant for 9 , but for only 1 did they equal or exceed -.70 . This indicates that an appreciable myocardial influence on PTT and SBP is evidenced when the sympathetic innervations are intact.

The DBP not only correlated less consistently with PTT but also was influenced by the pulse-wave recording site and type of stress. When recorded from the radial pulse wave, the correlations were significant and negative for 13 of 22 subjects but exceeded -.70 for only 2 . When recorded from the temporal and carotid pulse wave, the correlations were significant for 9 of 35 subjects, exceeding -.70 once. The variability of the correlations obtained between subjects from all pulsewave recording sites was also striking. For four subjects, the correlations were positive and significant. When the correlations were obtained for data from the cold pressor, the film, and associated rest periods separately from those for the avoidance task and associated rest periods, they were more negative under the former conditions. For example, the correlations based on the cold pressor and film data were significant for 30 of 55 subjects, exceeding -.70 for 5 . On the other hand, using only the data from the avoidance task, the correlations were less negative and, for 11 subjects were significant and positive. For five of the subjects, the correlations changed from significantly negative to significantly positive, the most extreme shift seen within a subject being from -.40 to +.82 . Finally, the effect of sympathetic blockade on the correlations between PTT and DBP was minimal.

\section{DISCUSSION}

The data from all six experiments concerning the

Table 1

Summary of Median Correlations (r) ${ }^{5}$ Between Blood Pressure (BP) and PTT Over All Conditions

\begin{tabular}{|c|c|c|c|c|c|c|c|c|c|c|c|c|}
\hline \multirow{3}{*}{$\begin{array}{c}\text { Pulse Site } \\
\text { BP Measure } \\
\end{array}$} & \multicolumn{8}{|c|}{ Intact Innervation } & \multicolumn{4}{|c|}{ Blocked Innervation } \\
\hline & \multicolumn{2}{|c|}{$\begin{array}{c}\text { Carotid } \\
\text { (Indirect) }\end{array}$} & \multicolumn{2}{|c|}{$\begin{array}{l}\text { Temporal } \\
\text { (Indirect) }\end{array}$} & \multicolumn{2}{|c|}{$\begin{array}{c}\text { Radial } \\
\text { (Indirect) }\end{array}$} & \multicolumn{2}{|c|}{$\begin{array}{c}\text { Radial } \\
\text { (Direct) }\end{array}$} & \multicolumn{2}{|c|}{$\begin{array}{c}\text { Carotid } \\
\text { (Indirect) }\end{array}$} & \multicolumn{2}{|c|}{$\begin{array}{c}\text { Radial } \\
\text { (Direct) }\end{array}$} \\
\hline & $\mathbf{r}$ & $\mathbf{N}$ & $\mathbf{r}$ & $\mathbf{N}$ & I & $\mathbf{N}$ & $I$ & $\mathbf{N}$ & $\mathbf{r}$ & $\mathrm{N}$ & $r$ & $\mathbf{N}$ \\
\hline $\begin{array}{l}\text { SBP } \\
\text { DBP }\end{array}$ & $\begin{array}{l}-.72 \\
-.01\end{array}$ & $\begin{array}{l}20 \\
15\end{array}$ & $\begin{array}{l}-.78 \\
-.17\end{array}$ & $\begin{array}{l}19 \\
18\end{array}$ & $\begin{array}{l}-.85 \\
-.30\end{array}$ & $\begin{array}{l}14 \\
15\end{array}$ & $\begin{array}{l}-.76 \\
-.44\end{array}$ & $\begin{array}{l}7 \\
7\end{array}$ & $\begin{array}{l}-.24 \\
-.07\end{array}$ & $\begin{array}{l}13 \\
13\end{array}$ & $\begin{array}{l}-.40 \\
-.09\end{array}$ & $\begin{array}{l}5 \\
5\end{array}$ \\
\hline
\end{tabular}

Note-Indirect refers to the noninvasive method of measuring blood pressure; direct refers to blood pressure measured invasively with a radial artery catheter. 
degree to which variations of PTT covary with blood pressure changes under the conditions of this study warrant the following conclusions. (1) PTT covaries with SBP appreciably in most individuals. These high correlations are observed whether SBP is measured indirectly or directly. When the sympathetic innervation of the heart is blocked, this degree of covariation is appreciably attenuated. (2) PTT covaries with DBP inconsistently. It tends to covary with DBP more appreciably and in a manner more similar to SBP when stressors are used which previous studies (Obrist et al., 1978) indicate evoke minimal sympathetic excitation on the heart and a more appreciable increase in total peripheral resistance. A more consistent and greater degree of covariation is also seen when the pulse wave is transduced furtherest from the heart. But, even at best, the consistency of the covariation as determined by the magnitude of the correlation coefficient is still not as appreciable as with SBP. Therefore, it appears that, under our experimental conditions, PTT provides an adequate measure of variations in SBP in most individuals but not in DBP. Both DBP and PTT are subject to several influences that can be independent, thus making the procedure of using PTT to index variations of DBP of dubious value unless these influences are known (see Obrist et al., Note 1 for a further discussion of this issue).

The results of this study are similar to those reported by Gribbin et al. (1976) and Steptoe et al. (1976). Because the Steptoe et al. study is more similar in procedural matters, some comparison of results is justified.

The magnitude of the correlations observed in the present study between SBP and PTT is slightly higher than in the Steptoe research. The basis for this is not entirely clear but may be explained by the fact that Steptoe used mean blood pressure whereby both DBP and SBP enter into the derived mean blood pressure value. However, Steptoe reported more appreciable correlations (i.e., all $>-.90$ ) when the data were grouped by plotting blood pressure in $2 \cdot \mathrm{mm} \cdot \mathrm{Hg}$ steps and obtaining the mean PTT for each step. This procedure, therefore, may have some merit. Steptoe made one other observation which exerts a note of caution in the use of PTT. With one of his stressors, namely, the inhalation of amylnitrate, a vasodilator, the correlations between mean pressure and PTT dropped essentially to zero. We have seen a similar effect at the initiation of the shock avoidance task. This was most obvious when blood pressure was directly recorded but was also evident in the other studies. What is observed is a marked decrease in PTT and a brief but sometimes pronounced decrease in both SBP and DBP. Usually, both SBP and DBP then recover in $15.30 \mathrm{sec}$, with SBP climbing to well above baseline, while DBP climbs very modestly to just above baseline. The duration of the effect is too brief to appreciably influence the relationship between PTT and SBP when evaluated with selected data points over all conditions. But this is clearly an indication that PTT and SBP can change in the opposite manner from that under other conditions.

Several procedural matters should be mentioned. First, the PTT measured in this study reflects two influences other than true PTT. ${ }^{2}$ At this stage, we cannot ascertain in definitive manner the extent of these influences, other than that they are appreciable. True PTT is defined as the duration for any one pulse wave between its appearance at one arterial site and its subsequent arrival at a more distal site. (Evidently, commercially manufactured equipment is available for this purpose.) We are not aware of any data on the validity and sensitivity of this procedure other than that presented by Gribbin et al. (1976), who measured true transit time on the arm. These investigators did not compare PTT to natural variations in blood pressure. Rather, they experimentally varied the arterial transmural pressure using a wide range of negative and positive pressures applied to the arm, and then ascertained the effects of this on transit time (which they converted to velocity). A pronounced relationship was found. Such a manipulation acts to vary the distensibility of the artery and thus alters the transit time, similar to the influence blood pressure has on the distensibility of the arteries. It is difficult to judge, even in light of the pronounced relationship they observed how sensitive transit time will be to natural variations in arterial distensibility and pressure. In the study by Steptoe et al. (1976), as in the present study, the PTT was triggered from the $R$ wave of the EKG, so the Steptoe data sheds no light on this issue.

A second procedural matter concerns the transduction of the pulse wave. This is always difficult, since it requires either a microphone or some other pressure-. sensitive transducer to be placed over an artery. As such, these transducers are sensitive to any variation in contact pressure with the skin which can result from movements and in turn produce a pulse wave that is not usable. The extent of such artifact can be considerable. We attempt to minimize such artifact by immobilizing the area from which the pulse wave is transduced. For example, when the radial pulse wave is used, both the hand and lower part of the arm are immobilized on a specially constructed device. In this way, sufficiently clean data are collected on each subject. We are currently exploring other methods to transduce a pulse wave that provides us reasonably clean data but without the necessity of immobilizing a limb or some other areas. Immobilizing an area is truly an encumbering technique under some circumstances and is not always practical.

In summary, the data from these experiments indicate an appreciable degree of covariation between PTT and SBP. If a reasonably artifact-free pulse wave can be transduced without encumbering the subject, then 
PTT may provide a reasonably sensitive and reliable means to track variations in SBP under certain conditions. Of course, PTT values as expressed in milliseconds have no correspondence to blood pressure values as expressed in millimeters $\mathrm{Hg}$ unless the PTT values on any one individual are calibrated against readings of the blood pressure in $\mathrm{mm} \mathrm{Hg}$.

In closing, one note of caution needs to be reemphasized. As previously indicated, the cardiodynamic determinants of PTT as measured in this study are still to be elucidated. Until this is done, one can use PTT only with reservations. For example, it may prove a more reliable index of SBP under some conditions than others. There remains a need to evaluate the sensitivity of true transit time. This measure may prove more sensitive to changes in DBP provided two artifactfree pulse-pressure signals can be obtained.

\section{REFERENCE NOTE}

1. Obrist, P. A., Light, K. C., McCubbin, J. A., Hutcheson, J. S., \& Hoffer, J. L. Pulse transit time: Relationship to blood pressure and myocardial performance. Manuscript submitted for publication, 1978.

\section{REFERENCES}

Gribin, B., Steptoe, A., \& Sleight, P. Pulse wave velocity as a measure of blood pressure change. Psychophysiology, 1976, 13, 86-90.

JeRnstedt, G., \& Newcomer, J. P. Blood pressure and pulse wave velocity measurement for operant conditioning of autonomic responding. Behavior Research Methods \& Instrumentation, 1974, 6, 393-397.

Obrist, P. A., Gaebelein, C. J., Teller, E. S., Langer, A. W., Grignolo, A., Light, K. C., \& McCubin, J. A. The relationship among heart rate, carotid $\mathrm{dP} / \mathrm{dt}$, and blood pressure in humans as a function of the type of stress. Psychophysiology, 1978, 15, 102-115.

Obrist, P. A., Lawler, J. E., Howard, J. L., Smithson, K. W., Martin, P. L. \& Manning, J. Sympathetic influences on cardiac rate and contractility during acute stress in humans. Psychophysiology, 1974, 11, 405-427.

Pickering, G. Personal views on mechanisms of hypertension. In J. Genest, E. Koiw, \& O. Kuchel (Eds.), Hypertension:
Physiopathology and treatment. New York: McGraw Hill. 1977. Pp. 598-605.

Steptoe, A., Smylan, H., \& Gribin, B. Pulse wave velocity and blood pressure change: Calibration and application. Psychophysiology, 1976, 13, 488-493.

Weltman, G., Sullivan, G., \& Gredon, D. The continuous measurement of arterial pulse wave velocity. Medical Electronics and Biological Engineering, 1964, 2, 145-154.

Williams, J. G. L.. \& Williams, B. Arterial pulse wave velocity as a psychophysiological measure. Psychosomatic Medicine, 1965, 27, 408-414.

\section{NOTES}

1. Nonencumbering refers to a procedure that permits the monitoring of blood pressure without requiring that the individual stop all activities; recording is done without the individual's apparent awareness, such as occurs with a sphygmomanometer and inflatable cuff. The latter method does not necessarily require that the individual stop all activities; under some conditions, such as with self-determination of blood pressure (Pickering, 1977), it is not known how representative a given self-determination is of the pressure just before the reading is taken. Whether self-administered or otherwise, with a cuff system, the individual is always aware of when the cuff is inflated. The effect of this on the existing level of blood pressure is unknown.

2. Two points need to be clarified. First, in some of the literature, reference is made to pulse-wave velocity. This is pulse transit time converted to velocity once the distance traveled is known. We do not make this conversion since our primary interests are intra-individual studies. Second, the method we use for measuring the transit time comprises the actual, or true, transit time, the pre-ejection period of the cardiac cycle and rise time of the pulse wave. This is necessitated by the methodology (see Obrist, Light, McCubbin, Hutcheson, \& Hoffer, Note 1 , for a discussion of this issue).

3. Mean arterial pressure is usually defined as the DBP plus one-third the pulse pressure.

4. A complete description of this system can be obtained by writing to the fourth author, J. Stanford Hutcheson.

S. A negative correlation between transit time and either SBP or DBP indicates that, as blood pressure is increasing, the PTT is decreasing. A decrease in the transit time would be expected as either cardiac performance increases or the distensibility of the arterial system decreases, as occurs with an increase in the peripheral resistance. 